\title{
The mechanisms of the protective effect of education in cognitive aging
}

\author{
Alena Sidenkova ${ }^{1, *}$, Vasilisa Litvinenko ${ }^{1}$, and Ilya Kalinin $^{2}$ \\ ${ }^{1}$ Ural State Medical University of the Ministry of Health of Russia, 620028, Repin Str., 3, \\ Yekaterinburg, Russian Federation \\ ${ }^{2}$ Ural Federal University named after the first President of Russia B.N. Yeltsin, 620002, Mira Str., 19, \\ Yekaterinburg, Russian Federation
}

\begin{abstract}
Frequent cases of pathological brain aging are an important scientific and social problem. Some people have the ability to compensate for the initial manifestations of pathological aging and delay the development of the clinical phenomena of the disease. The concept of "cognitive reserve" allows us to study the possibilities of increasing brain stability in conditions of pathological aging. The identification of the dominant form of thinking, converging or diverging, reveals possible neurophysiological mechanisms of the cognitive reserve. Understanding the mechanisms of formation of individual cognitive styles actualizes the contribution of the "Education" factor to the development of the cognitive reserve. The research material was scientific publications on the topic of work. It was revealed that the "education" factor can realize its protective effect on the pathological aging of the brain due to the formation of an adaptive form of thinking. The data presented in this review make it possible to substantiate the medical and social significance of educational programs for people of mature and older age.
\end{abstract}

\section{Introduction}

Older people are increasingly seen as active participants in the process of social development of modern society, this determines the need to maintain high subjective activity of the elderly person and high cognitive abilities [1]. The participation of an aging person in social and production processes is limited by cognitive disorders, which are an example of pathological aging of the central nervous system. Most cases of cognitive impairment are progressive in nature with a high risk of dementia [2,3]. The results of individual studies show that in some people with amniotic type MCI with a high concentration of amyloid in the brain it does not correlate with a decrease in their functional and social activity $[4,5]$. That is, the risk of transforming mild cognitive impairment (MCI) into dementia is not fatal.

Thus, the increasing involvement of older people in production and social processes determines the need to maintain their high cognitive tone, which increases the relevance of

\footnotetext{
* Corresponding author: sidenkovs@ mail.ru
} 
research related to the study of biological reserves of the brain and psychological, social mechanisms of adaptation of an elderly person.

\section{Materials and Methods}

The material of the study was scientific publications on the topic of work.

The general scientific method was applied: analysis of modern scientific literature on the problem of research, generalization, comparison, systematization of theoretical data on factors contributing to the preservation of normative parameters of cognitive functions in the elderly.

\section{Results and Discussion}

Scientists describe the phenomenon of brain resistance and its function in connection with age-related diseases. Some researchers compared the results of post-mortem histological tests and intravital clinical observations and found that elderly people with a large brain volume and a large number of neurons compared with age-comparable people did not have clinical manifestations of cognitive impairment during their lifetime. It has been suggested that these patients had a large "stock" of neurons and abilities that allowed them to compensate for losses caused by Alzheimer's disease [6]. The concept of "cognitive reserve" (CR) was put forward taking into account the individual characteristics of the vulnerability of the brain to age-related and pathological changes (for example, to Alzheimer's disease) [7]. Some authors indicate that a person's susceptibility to the development of brain pathology is the result of the interaction of multidirectional processes: damage to brain tissue and the ability of the brain to maintain high functional activity due to "brain reserve" (BR) [8]. The followers of this opinion believe that the calculated indicator of the "brain reserve" is the totality of the "quantitative" parameters of the brain: the total size of the brain, the number of neurons, synapses, density of dendrites, etc. [9]. This interpretation of the concept of "brain reserve" considers BR as a model of a passive threshold, which predicts that after a certain quantitative ratio of pathological changes in the central nervous system is reached, brain functioning will inevitably deteriorate. Some authors describe the "cognitive reserve" (CR) in the framework of the active model, indicating that the threshold for functional decline is determined not by quantitative measurements of the brain, but by a set of changes determined by human experience [10]. From these positions, CR is defined as a brain resource that develops throughout life, stimulates cognitive activity and protects a person from clinical signs of cognitive decline [11]. Thus, the concepts of "brain reserve" and "cognitive reserve" make an independent and synergistic contribution to understanding individual differences in the resistance of brain functions to brain pathology [12].

Followers of the concept of "active cognitive reserve" believe that the reserve is determined not by the volume of the brain, but by the quality of neural networks. Wide neural networks allow you to efficiently process information, eliminating damaged links from your chain. According to Y.Stern, the cognitive reserve is the ability of the brain to optimize or maximize its performance due to the differentiated set of neuronal connections, which leads to the use of alternative cognitive strategies [13]. Modern researchers are actively studying the psychophysiological mechanisms of CR and the factors that influence its formation. Existing data confirm the fact that $\mathrm{CR}$ acts as a protective mechanism of cognitive impairment and as a factor that increases potential of to restore cognitive function in the case of disorders $[14,15]$. 
The reserve is the individual variability of the functional or structural integrity of the nervous system, which changes the cognitive and behavioral abilities of a person in case of brain pathology [16]. Understanding of this mechanism is promoted by the theory of the global working space of the brain. According to this theory, the entire neuronal system of the brain is a universal computing environment in which different tasks are assigned to different areas, but in the absence of resources the brain can transfer this task to another area. The result is a map of brain activity that corresponds to various tasks. With the dominance of convergent thinking, the main attention is paid to the recognition of already familiar information, ways to reuse it and increase the amount of stored information. With the dominance of convergent thinking, the left hemisphere and interhemispheric interference of information is weakened, which is associated with the insensitivity of neurons to change their state when signals are received at their inputs (dendrites). With pathological aging of the brain, aggregates of beta-amyloid destroy synapses. In Alzheimer's disease, it was proven that peptide oligomers interact with CamKII protein kinase and disrupt its normal mobilization. The main function of CamKII is disrupted control of the plasticity of neurons and increased response to received signals. The synaptic connection between the neurons responsible for storing information is broken. Amyloid beta prevents the participation of the CamKII molecule in the activation of the synapse, leads to degradation of the synapse and reduces the ability of this neuron to participate in the transmission of information [17]. In the initial stages of the development of Alzheimer's disease, the ability of the brain to compensate for the disease remains. When convergent thinking dominates the subject, the inherent style of interaction with information excludes the possibility of activating associative collaterals, which leads to cognitive deficiency with a small number of painful brain lesions. The presence of divergent thinking allows a person to evaluate several creative ideas at once, to make non-standard decisions. The dominance of divergent thinking correlates with an increase in the volume of the right dorsolateral prefrontal, right lower dark cortex. The degree of integral white matter of the brain (an indicator of fractal anisotropy) according to the results of diffuse-tensor images positively correlates with creativity. It is proved that the higher the index of fractal anisotropy, the brighter the white matter of the brain and the better the index of myelination. The paths of white matter facilitate the creative process through a sufficient integration of information and various higher cognitive functions, such as working memory, constant attention, generation of ideas, cognitive flexibility, which are vital for overcoming old ideas and developing new models of thinking [18]. In the process of ontogenesis, the formation of active synapses, dendrites and their myelination is replaced by the epigenetically determined presence of neuronal efferent compounds. This second process is due to the active influence of the environment and the subject's activity of the individual. The formation of the dominant form of thinking and individual cognitive style is formed throughout the life of the individual. Education is the most significant factor influencing the dominant form of thinking.

Education factor. One of the often mentioned protective factors traditionally assessed in screening tools aimed at detecting dementia is "education" [19]. According to the hypothesis of Ya.A. Mortiner (1988), education provides protection against age-related dementia by increasing the "cognitive reserve" of the brain [20]. Studies conducted in different countries (Brazil, China, Finland, France, Italy, Israel, Sweden, USA), confirmed this hypothesis [21].

Epidemiology. A meta-analysis of 28 cohort studies revealed a 1.72-fold risk of dementia (95\% CI: $1.52-1.96)$ in people with a low level of education and a $40 \%$ reduction in the risk of dementia at a high educational level [22]. Longitudinal studies of the risk of dementia revealed an increase of 2.2 times in people with an education level of less than 8 years and 2.25 times with a low professional level compared to people with a higher 
education level or a higher professional level. Epidemiological studies of Arenaza-Urquijo E. et al. They showed a lower prevalence / incidence of dementia in older people with a high level of education [23].

Clinical signs. Having established that a high level of education, which determines the cognitive reserve, is associated with a reduced risk of cognitive impairment even in people with a high-risk genetic background (for example, carriers of E4 apolipoprotein), C. Phillips (2017) examined the problem of the relationship between the "educational" factor and the reserve $\mathrm{CR}$ in a new plane, suggesting that the quality and level of education received change the "threshold" of the reserve, increasing it, while simultaneously determining the "collapse" of a high cognitive reserve with a large accumulation of pathological mother ala in the central nervous system [24]. This allows us to admit an understanding of the "cognitive reserve" as a certain quantitatively measurable quantity with a limited "volume". For example, the work of A. David et al. (2014) demonstrated the protective effect of education on cognitive preservation only at an early stage of atrophic disease. The authors observed a paradoxical effect of the "educational level" factor in moderate and severe Alzheimer's disease, which was opposite to expectation. It has been suggested that education is associated with a slower rate of cognitive decline before disease, but a faster rate of cognitive decline after the occurrence of dementia. Upside down indicates the complexity of the relationship between the "educational" factor and the "cognitive reserve", since education does not completely delay the onset of cognitive decline, moreover, it is associated with accelerated progression of cognitive loss in cases where cognitive decline is phenomenologically represented [28]. The information provided requires clarification of the features of education and other intellectual activities of persons participating in such studies. The relationship between the quality of educational activities and cognitive functions was observed only in participants with 12 years of education. This suggests that education outside high school can soften the link between lower quality education and lower cognitive testing effectiveness, which indicates the importance of stimulating cognitive activity throughout life, which protects against phenomenologically manifested cognitive decline [25].

The concept of cognitive reserve explains the results of numerous studies, which show that the level of intelligence, general and professional education is a predictor that brain damage should be significant enough before a functional deficit appears. The cognitive reserve hypothesis states that the brain in individuals with a large cognitive reserve processes tasks more efficiently.

The cognitive reserve model refutes the claim that there is a certain fixed threshold for the number of neuronal synapses, beyond which a functional deficit develops. This critical threshold differs in different people, depending on how efficiently the preserved neural substrate is used.

Compensatory potential of the "education" factor. The British large-scale populationbased study of cognitive function and aging, lasting 12 years, evaluating the Cognitive Lifestyle Score (CLS), as a combination of cognitive activity (education, professional complexity) and social activity at different periods of life, revealed that a higher level CLS protected against dementia (odds ratio $=0.6,95 \%$ confidence interval: $0.4,0.9$ ). The sensitivity analysis showed that this main effect was reliable and reproducible, taking into account at least 2 components of the assessment: "education" + "occupation" or "education" + "late age" [26]. The insufficiency of children's education for the subsequent development of intelligence and protection from dementia was indicated by N. Scarmeas et al. (2003), revealing that maintaining intellectual activity during the subsequent life is an important factor in the formation of CR [27].

The value of cognitive activity in adulthood. The study showed that age-related cognitive impairment is especially pronounced in cognitive (executive or executive) control 
impairments that are associated with "fluid intelligence" indicators, such as a person's ability to find non-standard solutions to familiar problems. The presence of a large number of diverse experience data ("higher education" and "intellectual wealth of activity" during life) correlates with an increased level of "fluid intelligence" and qualitatively cognitive functioning in old age [28]. Opinions about the mechanism of maintaining the activity of performing functions in the process of an active cognitive process are different. The results of neural connections, the discovery that the number of dendrites negatively correlates with the results of an intelligence test. This is an unambiguous assumption that in the process of educational and cognitive activity of a person throughout his life there is a kind of "selection" of qualitative neuronal connections [29]. Probably, not all education contributes to the formation of a cognitive reserve. The use of tasks of various types in the educational process increases the divergent mental possibilities and creative abilities of a person, while formalized indicators of intelligence can remain at the same level. But the developing paths of the white matter of the brain, which facilitate the creative process through a sufficient integration of information and various higher cognitive functions, such as working memory, constant attention, generation of ideas, cognitive flexibility, which are vital for overcoming old ideas and developing new models of thinking, increase cognitive reserve. In the study of neurochemistry "creative brain", the concentration index of N-acetylspartate (NAA) was used. This metabolite is involved in many physiological processes - in the transport storage of aspartate and glutamate, in the formation of myelin and is a marker of the health of neurons [30]. In the works of Russian authors, it was shown that the choice of a nonstandard solution is characterized by the presence of a front-right focus of maximum activation at rest, while the left semantic region of Wernicke is strongly activated during the solution. It is wrth recalling that this particular area of the brain is most vulnerable to pathological aging, in particular for Alzheimer's disease. Divergent thinking is accompanied by an increase in the "spatial complexity of the EEG" (that is, the number of cell ensembles simultaneously involved in the work), which reflects, firstly, the destruction of a stable mental associative habit and the extension of the activation region to previously inactive ones, and secondly, a higher degree of freedom in the interactions of cortical neural ensembles [31]. Creative behavior causes an increase in alpha synchronization, which correlates with the performance of various cognitive tasks related to the involvement of episodic short-term memory, visual-spatial working memory, and selective inhibition of unnecessary information during visual-spatial orientation [32]. Persons with pronounced divergent thinking when solving problems have higher rates of cerebral blood flow in the prefrontal and frontotemporal regions than those with a convergent type of thinking. In the study of glucose metabolism in solving problems of verbal fluency, a negative correlation of results was shown [33].

\section{Conclusion}

Thus, studies show that the mechanisms of formation of the cognitive reserve are diverse. Active cognitive reserve is formed in the process of educational and cognitive activity of the subject. The nature of educational activities affects the formation of the dominant form of thinking. Neurophysiological, neurochemical studies have proved that cognitive activity based on the participation of divergent thinking makes a significant contribution to enhancing the cognitive reserve. 


\section{References}

1. World Population Prospects: The 2017 Revision. Department of Economic and Social Affairs Population Division. ESA/P/WP/248. (United Nations, New York, 2017) URL: https://population.un.org/wpp/

2. L. Clare, Y-T. Wu, J.C. Teale, C. MacLeod, F. Matthews, C. Brayne, PLoS Med, 14(3), e1002259, (2017) doi:10.1371/journal.pmed.1002259

3. B.A. Lautarescu, A.J. Holland, S.H. Zaman, Neuropsychol. Rev., 27(1), 31-45, (2017) doi: 10.1007/s11065-017-9341-9

4. T. Annus, L.R. Wilson, Y.T. Hong, J. Acosta-Cabronero, T.D. Fryer, A. CardenasBlanco, Alzheimers Dement., 12(5), 538-45, (2016) doi:10.1016/j.jalz.2015.07.490

5. A. DiCarlo, M. Baldereschi, L. Amaducci, et. al., J. Am. Ger. Soc., 48, 775 - 782 (2000)

6. J.D. Medaglia, F. Pasqualetti, R.H. Hamilton, S.L. Thompson-Schill, D.S. Bassett, Neurosci Biobehav Rev., 75, 53-64, (2017) doi: 10.1016/j.neubiorev.2017.01.016.

7. R.N. Jones, T.G. Fong, E. Metzger, S. Tulebaev, Am. J. Geriatr. Psychiatry, 18(2), 117-127 (2010) doi: 10.1097/JGP.0b013e3181b972e8

8. D. Barulli, Y. Stern, Trends Cogn Sci., 17(10), 502-9 (2013) doi: 10.1016/j.tics.2013.08.012

9. A. Bhat, J. Biosci., 40(2), 205-8 (2015) doi:10.1007/s12038-015-9511-y

10. D. Cadar, A. Robitaille, S. Clouston, S.M. Hofer, A.M. Piccinin, G. Muniz-Terrera, Neuroepidemiology., 48(1-2), 9-20 (2017) doi: 10.1159/000452276

11. M. Perquin, N. Diederich, J. Pastore, M.L. Lair, S. Stranges, PLoS ONE., 10(9), e0138818 (2015) doi:10.1371/journal. pone.0138818

12. J. Steffener, Y. Stern, Biochim. Biophys. Acta., 1822(3), 467-73, (2012) doi: 10.1016/j.bbadis.2011.09.012 URL: https://www.ncbi.nlm.nih.gov/pubmed/21982946

13. Y. Stern, J. Int. Neuropsychol. Soc., 8(3), 448-60 (2002)

14. Ko Kang,Soo Byun Min, Yi Dahyun, Ho Lee Jun, Hyung Kim Can, Young Lee Dong, Front. Aging Neurosci., 10, 70 (2018) doi: 10.3389/fnagi.2018.00070

15. O. Yu. Strizhitskaya, Vestn. S.-Peterb. un-ta, 16, (2016) doi:10.21638/11701/spbu16. 2016.209

16. N.N. Koberskaya, G.R. Tabeea, Neurology, Neuropsychiatry, Psychosomatics, 11(1), 96-102 (2019) doi:10.14412/2074-2711-2019-1-96-102

17. D.J. Poulsen, D. Standing, K. Bullshields, K. Spencer, P.E. Micevych, A.M. Babcock, Journal of Neuroscience Research, 85(4), 735-739 (2007) doi:10.1002/jnr.21163

18. J. Cohen-Adad, M. Descoteaux, S. Rossignol, R.D. Hoge, R. Deriche, H. Benali, Neuroimage, 42(2), 739-749 (2008) doi:10.1016/j.neuroimage.2008.04.243

19. M. Crowe, O.J. Clay, R.C. Martin, V.J. Howard, V.G. Wadley, P. Sawyer, R.M. Allman, J. Gerontol. A Biol. Sci. Med. Sci., 68(2), 198-204 (2013) doi: 10.1093/gerona/gls122

20. J. Mortimer, Adler Intellect: Mind over Matter, 205, (Macmillan Publishing Company, 1990).

21. J.A. Mortiner, Do psychosocial risk factors contribute to Alzheimer's disease? A.S. Hendersen, J.H. Hendersen (Eds), Etiology of dementia of Alzheimer's type, 39-52, (1988)

22. Dementia and Risk Reduction. An analysis of protective and modifiable factors. World Alzheimer Report, (2014)

23. E.M. Arenaza-Urquijo, B. Landeau, R. La Joie, K. Mevel, F. Mézenge, A. Perrotin, B. Desgranges, D. Bartrés-Faz, F. Eustache, G. Chételat, Neuroimage., 83, 450-7, (2013) doi: 10.1016/j.neuroimage.2013.06.053.

24. C. Phillips, Neural. Plast., 3589271, (2017) doi: 10.1155/2017/3589271 
25. David A. Bennett, Steven E. Arnold, Michael J. Valenzuela, Carol Brayne, Julie A. Schneider, Acta Neuropathol., 127(1), 137-150 (2014) doi:10.1007/s00401-013-12262.1

26. M. Valenzuela, C. Brayne, P. Sachdev, G. Wilcock, Amer. Journ. of epidem., 9, 1004$1012(2011)$

27. N. Scarmeas, E. Zarahn, K. Anderson, et al, Arch. Neurol., 60(3), 359-65 doi: http://dx.doi.org/10.1001/archneur.60.3.359

28. B.B. Velichkovskiy, Eksperimental'naya psikhologiya. 3, 78-91 (2009) URL: http://psyjournals.ru/exp/2009/n3/24925_full.shtml

29. A. M. Tucker, Y. Stern, Current Alzheimer research, 8(4), 354-360 (2001)

30. R.A. Chávez-Eakle et al, Neuroimage, 38, 519-528 (2007)

31. M.G. Starchenko, The brain organization of verbal creative thinking (St. Petersburg, 2018)

32. E.N. Benderskaya, K.V. Nikitin, SPbSPU Scientific-Technical Journal, 6, 34-40 (2011)

33. H. Budde, M. Wegner, The Exercise Effect on Mental Health: Neurobiological Mechanisms, 1st Edition, 518 (CRCPress, 2018)

34. A. Eskes, Changing Brains: Applying Brain Plasticity to Advance and Recover Human Ability. Progress in Brain Research, 207 (Elsevier, 2013)

35. K. Hilger, M. Ekman, C.J. Fiebach, U. Basten, Scientific Reports, 7(1) (2017) doi: 10.1038/s41598-017-15795-7) 